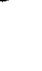

\footnotetext{
Intrabeam Scattering Results for a
}

High"Frequency RF System with Tight"RF Buckets -

\section{G. Parzen}

May 25, 1988 
(1)

Factors Leading To Growth

1) $\frac{1}{\sigma} \frac{d \sigma}{d t} \simeq\left(Q^{2} / A\right)^{2}$

2) Distance from "Equilibrium State"

Invariant $\Rightarrow \sigma_{E}^{2}-\sigma_{X}^{2}=$ cons $\quad \sigma_{E}=\bar{X}_{p} \sigma_{p}$ $\gamma \geq \gamma_{t}$, cells only lattice, ho coupling

$$
\begin{aligned}
t \rightarrow \infty & \sigma_{E} \sim \sigma_{x} \\
\frac{1}{\sigma_{E}} \frac{d \sigma_{E}}{d t} & =\left(\frac{\sigma_{x}}{\sigma_{E}}\right)^{2} \frac{1}{\sigma_{x}} \frac{d \sigma_{x}}{d t}
\end{aligned}
$$

If $\sigma_{E} \ll \sigma_{x}$ at $t=0$, the $\sigma_{E}$ will grow much faster than $\sigma_{x}$ until $\sigma_{E} \sim \sigma_{x}$.

Protons may show large growth in $\sigma_{E}$, if $\sigma_{E}<\sigma_{x}$ at $t=0$. 
(2)

High Frequency RF System

To get shorter $\sigma_{2}$, suggests $f \sim 200 \mathrm{MHz}$

Earlier calculations $r$ required

large voltage $V \cong 4_{0} m \bar{V}$, and gave

large growth in $\varepsilon_{x}, \sigma_{p}$, by factor $\simeq 2$.

Old Procedure

$V$ held constant. V chosen

large enough to contain butch

at $\gamma=100 j \Delta_{B}=2.5 \sigma_{p}$ at $\gamma=100$ after lo hours.

New Suggested Procedure

$V$ varied with time so that bucket just contains the bunch, $\Delta_{B}=2 \sigma_{p}$ at all times.

For_fixed $\sigma_{p} / \Delta_{B}, \sigma_{l}$ is constant.

$$
\frac{\sigma_{p}}{\Delta_{B}}=\sin \phi / 2
$$

For $\Delta_{B}=2 \sigma_{P}, \phi=60^{\circ}=\frac{1}{3} \pi$

$$
\sigma_{l}=\frac{1}{3} l_{B}
$$

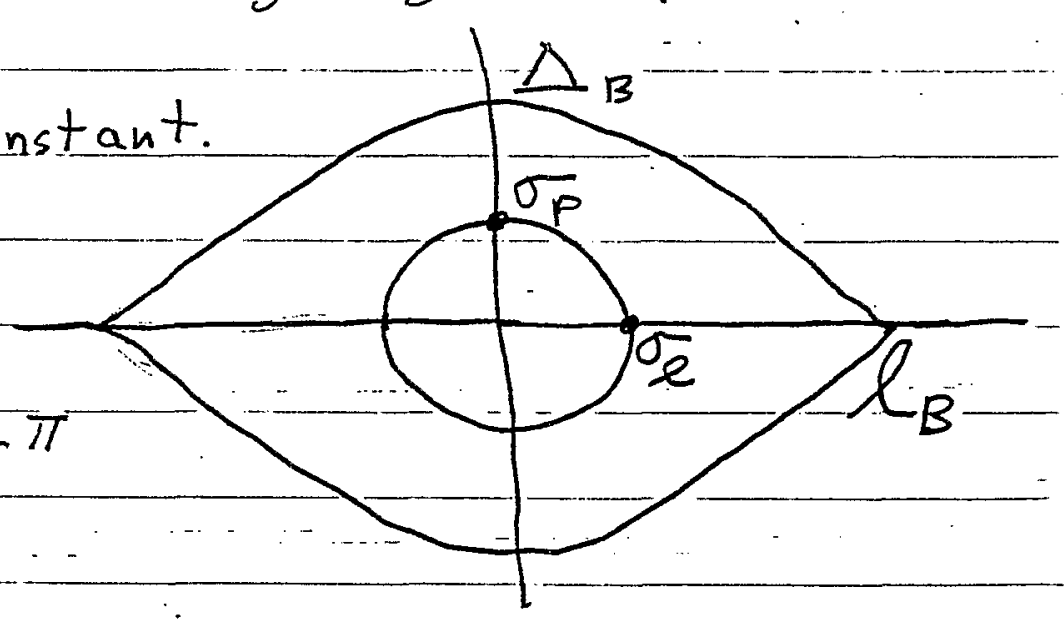


(3)

Proposed RF System

$$
\begin{aligned}
& f=160 \mathrm{MHz}, \quad h=2052 \\
& \sigma_{l}=31 \mathrm{~cm}, \quad A=.3 \mathrm{eV}-2 e c \\
& \triangle_{B}=2 \sigma_{P}
\end{aligned}
$$

i) Increasing $f$ will reduce $\sigma_{l}$ but will increase $\varepsilon_{x}, \sigma_{p}$ and $V$

2) Increasing A will increase

$$
\varepsilon_{x,} \sigma_{p} \text {, and } V \text {. }
$$

3) Increasing $\Delta_{B} / \sigma_{p}$ will reduce $\sigma_{e}$ and improve the safety (reduce the losses from the bucket) but will increase $\varepsilon x, \sigma_{p}$ and $V$ 
$\therefore \quad$ Intrabeam Scattering, 10 hrs - Giparzen, 5124188

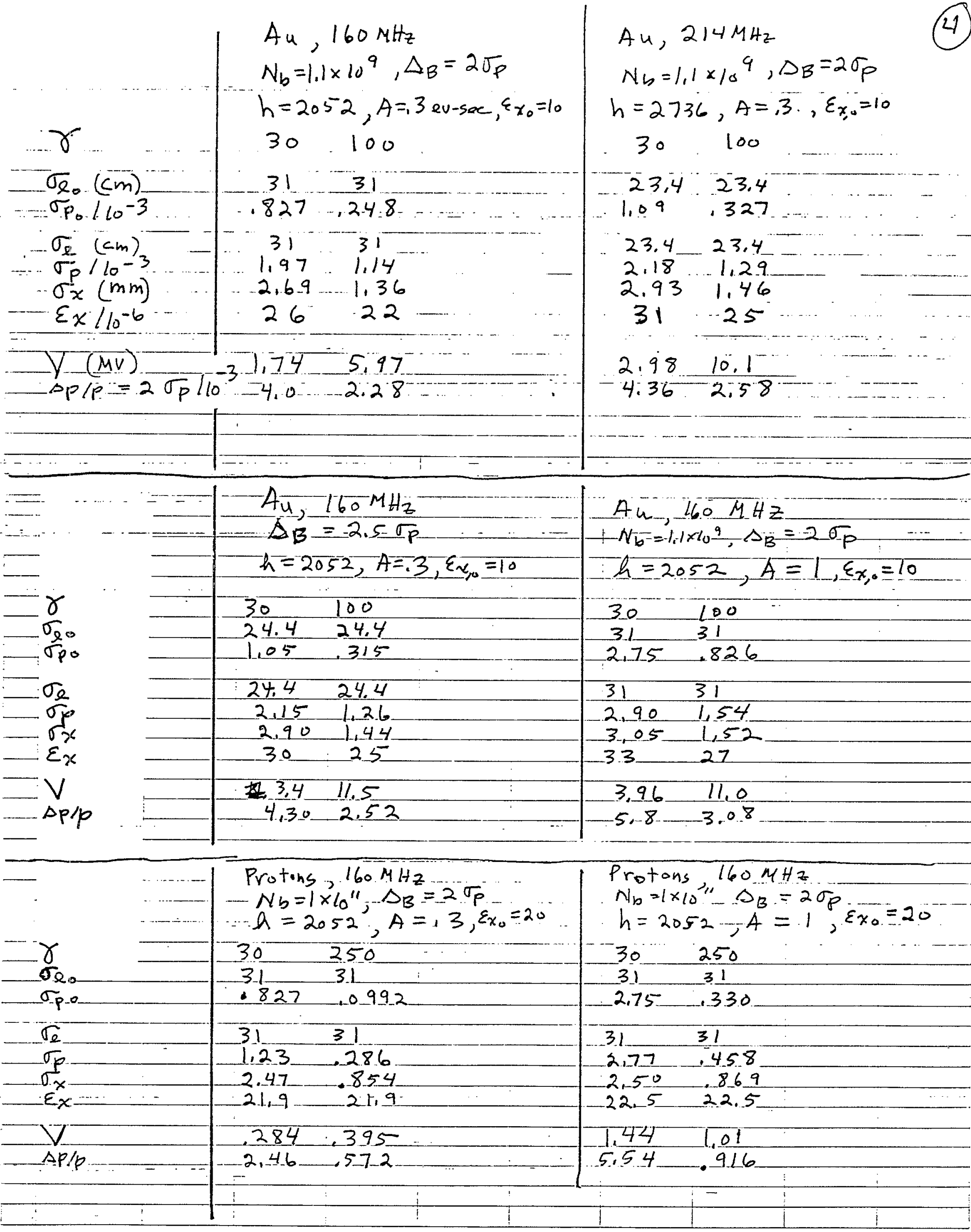




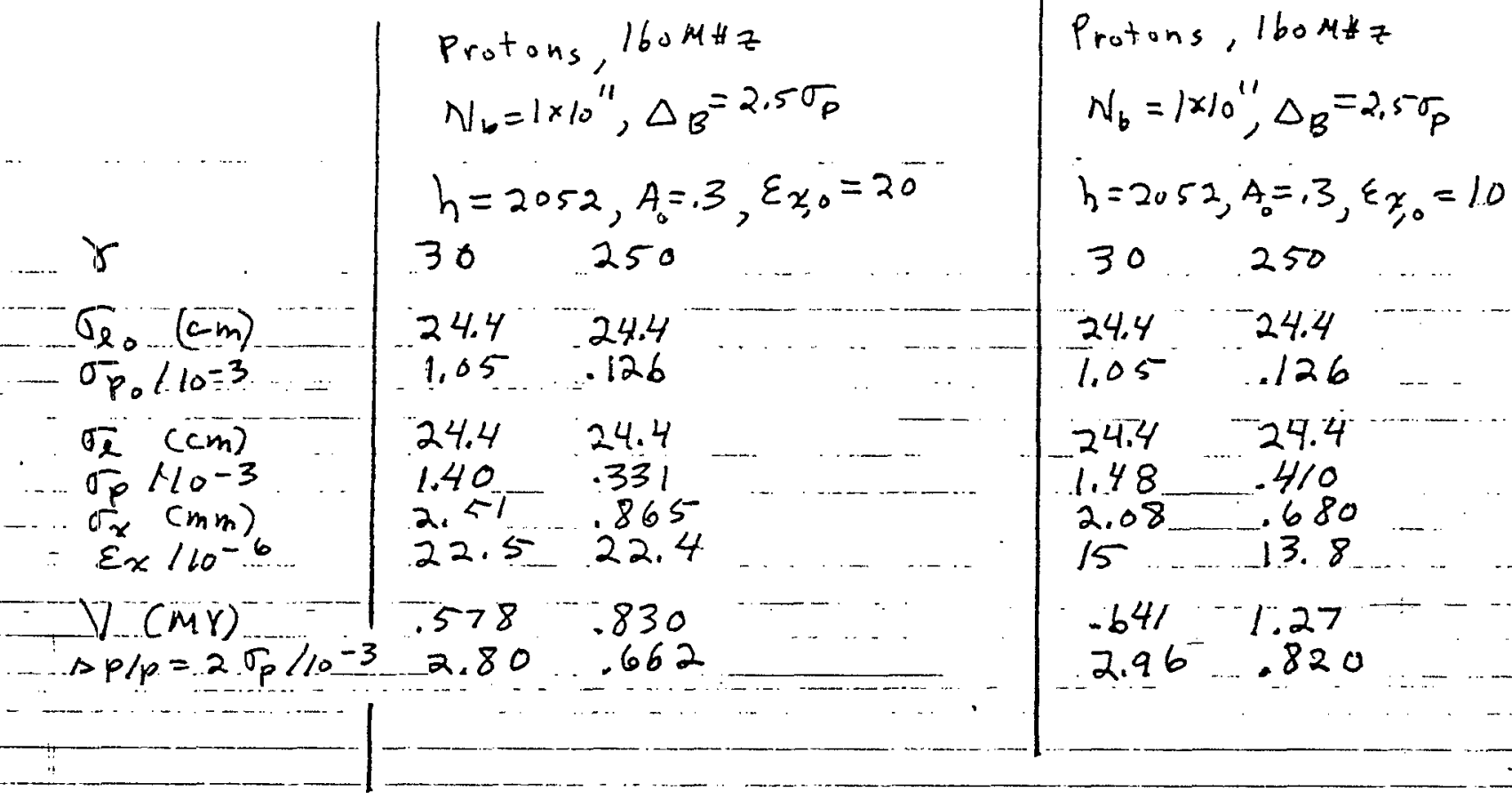




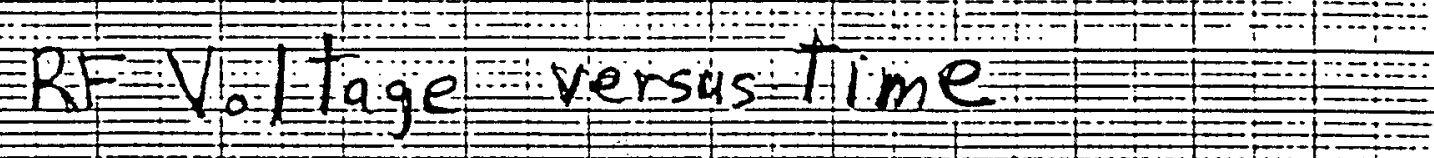

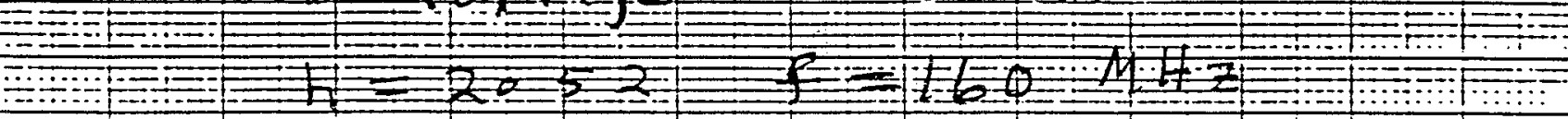
닐

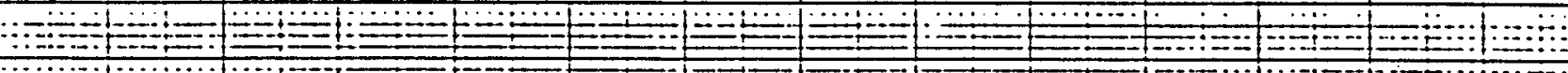

命

$>$

$4 \triangle_{B}-2 T_{P}, \varepsilon_{0}=20, \gamma=250$

3

2

.1

年

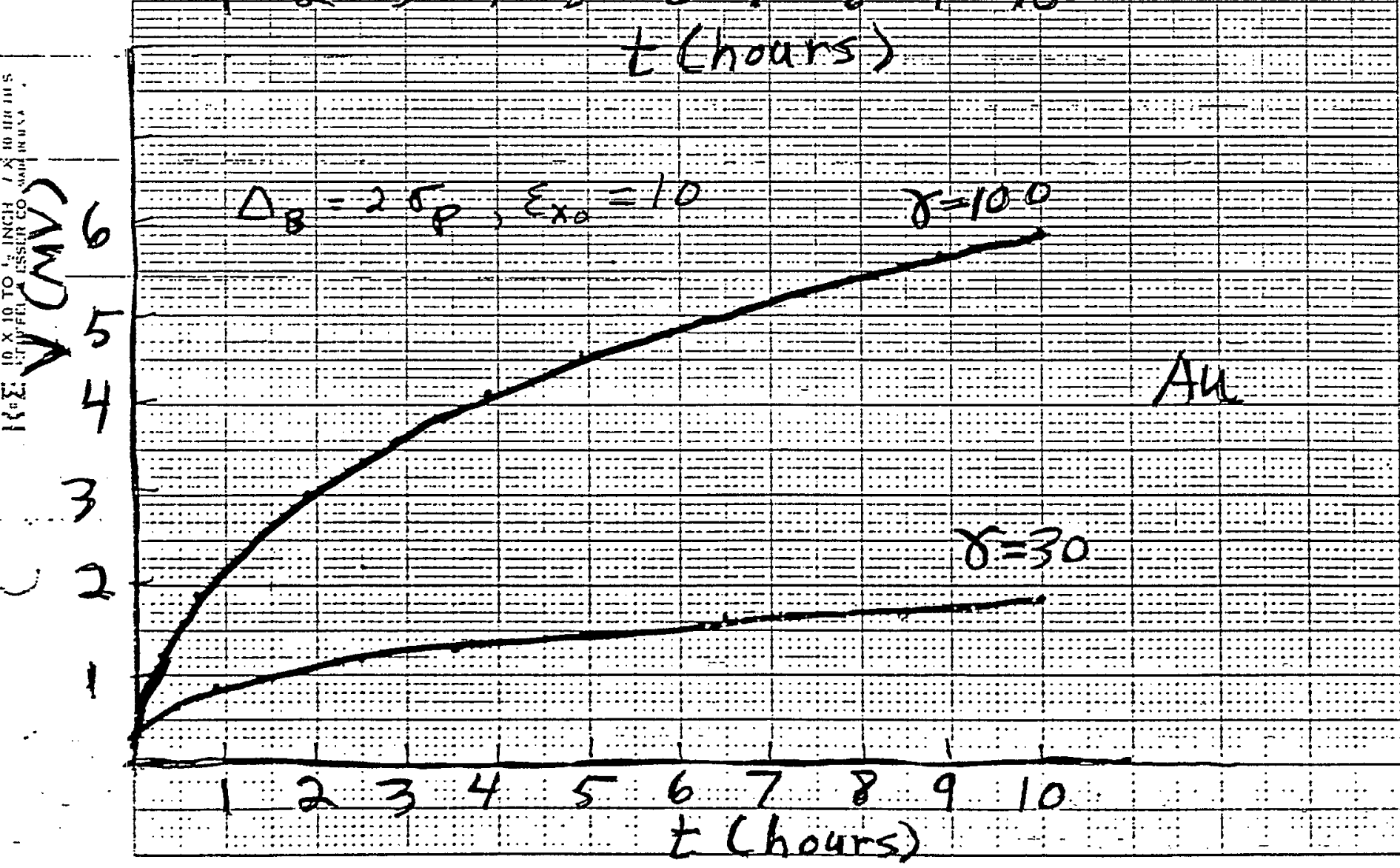

